\title{
Phosphate Control in Chronic Kidney Disease: an Unresolved Issue
}

\author{
Antonio Bellasi ${ }^{1}$, Luca Di Lullo ${ }^{2}$, Biagio Di Iorio ${ }^{3}$ and Domenico Russo ${ }^{4}$ \\ ${ }^{1}$ UOC di Nefrologia, Ospedaliera Sant'Anna, Italy \\ ${ }^{2}$ Department of Nephrology and Dialysis, Ospedale Parodi, Italy \\ ${ }^{3}$ Department of Nephrology and Dialysis, PO "A Landolfi" - Solofra, Italy \\ ${ }^{4}$ Department of Nephrology, University "Federico II" Napoli, Italy
}

Received: May 31, 2018; Published: June 15, 2018

*Corresponding author: Antonio Bellasi, MD, PhD, Department of Nephrology and Dialysis, Ospedale Sant'Anna, ASST-Lariana, Italy

Keywords: Phosphate Balance; Phsophorous; Phosphate Binder; Chronic Kidney Disease; Serum Phosphate; Numerous kinases; Normophosphatemia; Phosphate Homeostasis Perturbations; Placebo; Lanthanum Carbonate; Sevelamer Carbonate; Calcium Acetate; Plasma; Hyperphosphatemic

Abbreviations: ESRD: End Stage Renal Disease; CKD: Chronic Kidney Disease; ATP: Adenosine Triphosphate; DNA: Deoxyribonucleic Acid; PTH: Parathyroid Hormone; FEP: Fraction Excretion of Phosphate; RCT: Randomized Clinical Trial; FGF: Fibroblast Growth Factor

A significant body of evidence supports the notion that higher levels of serum phosphate are associated with poor survival [1-3]. Similar outcome data have been reported in end stage renal disease (ESRD) as well as individuals with various degree of renal function impairment [1-3]. However, the lack of randomized clinical trial (RCT) and a certain degree of heterogeneity among different studies in this domain preclude the definition of what is the optimal range of serum phosphate to target in different chronic kidney disease (CKD) stages [1-3]. Phosphorus is an essential element for life. It is a key factor in energy metabolism being a fundamental constituent of adenosine triphosphate (ATP) [2]. Similarly, phosphate is a core element of deoxyribonucleic acid (DNA) as well as a cell membranes. Numerous kinases and enzymes utilize phosphate for signal transduction and regulation of various metabolic pathways [3]. Although fundamental, several lines of evidence suggest that in some conditions phosphate may trigger unwanted effects.

Although mechanisms are far from being elucidated, in vitro and in vivo evidence suggest that phosphate homeostasis perturbations are associated with endothelial dysfunction [4], arterial stiffness [5], left ventricular hypertrophy [6], carotid artery intima media thickness [7], vascular calcification [8], infection that in turn may explain the astonishing risk of all-cause as well as CV mortality or CKD progression associated with increased serum levels of phosphate. Of interest, the observed relationship between phosphatemia and outcome is almost linear in CKD [9] and J-shaped in ESRD [10], suggesting that the relative risk associated with similar serum levels may change in different stages of renal function impairment or according to confounding factors such as malnutrition. In light of these uncertainties, ad hoc RCT to determine the optimal range of serum phosphate to aim for in clinical practice would be needed. Preliminary studies that investigated the impact of phosphate modulation on surrogate outcomes, however, have been disappointing [11,12]. Indeed, administration of full doses of different phosphate binders had only trivial effects on serum levels of phosphate and no or detrimental impact on some surrogate outcome $[11,12]$.

Chue and coworkers [11], tested the impact of sevelamer Vs placebo on left ventricular cardiac mass in a cohort of 109 subjects with normophosphatemia and CKD stage 3. At study completion, no effect on serum levels of phosphate or cardiac mass was reported [11]. In another small RCT, Block and coworkers [12], assigned 
148 CKD stage 3-4 subjects with normal serum levels of phosphate to either placebo, lanthanum carbonate, sevelamer carbonate or calcium acetate [12]. At the end of the 9-months follow-up significant reduction in phosphaturia coupled by minimal effects on phosphatemia were observed [12]. Remarkably, treated subjects showed a worrisome trend toward coronary artery calcification progression among subjected treated with calcium acetate [12]. While the first observation suggest that serum levels may be poorly associated with phosphate balance, the second observation questions the use of phosphate binders in normophosphatemic CKD patients.

Whether is serum phosphate levels or balance what modulate the risk associated with phosphorous metabolism is an ongoing debate [1]. However, compelling evidence suggest that chronic exposure to high phosphate intake and positive balance triggers the release of phosphaturic factors such as fibroblast growth factor 23 (FGF23) and parathyroid hormone (PTH) [2]. In the Heart and Soul study cohort, Dominguez and collaborators [13] found that FGF23 and fraction excretion of phosphate (FeP) modulated the risk of all-cause mortality as well as CV events in a cohort of 872 subjects with only minimal renal function impairment. Of interest, higher FeP mitigated the risk burden associated with elevated FGF23 suggesting that an efficient renal excretion of phosphate decreases the risk burden associated with positive phosphate balance [13]. Other pieces of evidence support the notion that phosphate burden induces renal fibrosis and mitigate the renoprotective effect of Ramipril [14] as well as low protein diet [15].

The relatively poor correlation between phosphate levels and balance maybe explained by the intracellular or interstitial distribution (99\% of the total body pool) of this anion and the complicated interplay between the intracellular and the intravascular compartments that determine the amount of phosphate is easily accessible for both renal or hemodialysis clearance [1,2]. Balance study performed in chronic hemodialysis patients suggest that phosphate clearance is a linear function of time while serum levels exhibit a 2 phases profile during dialysis (Figure 1) [16] and it has been postulated that phosphate mobilization from extravascular compartments may prevent excessive reduction of phosphate concentration in plasma and intracellularly $[17,18]$. Hence, serum levels maybe function of several other factors than phosphate balance (Figure 2) [17,18]. If studies on normophosphathemic patients have failed to demonstrate any meaningful impact of phosphate binders on surrogate endpoints, [11,12] few studies in hyperphosphatemic patients yield promise in term of the potential impact phosphate balance metabolism $[4,19]$.

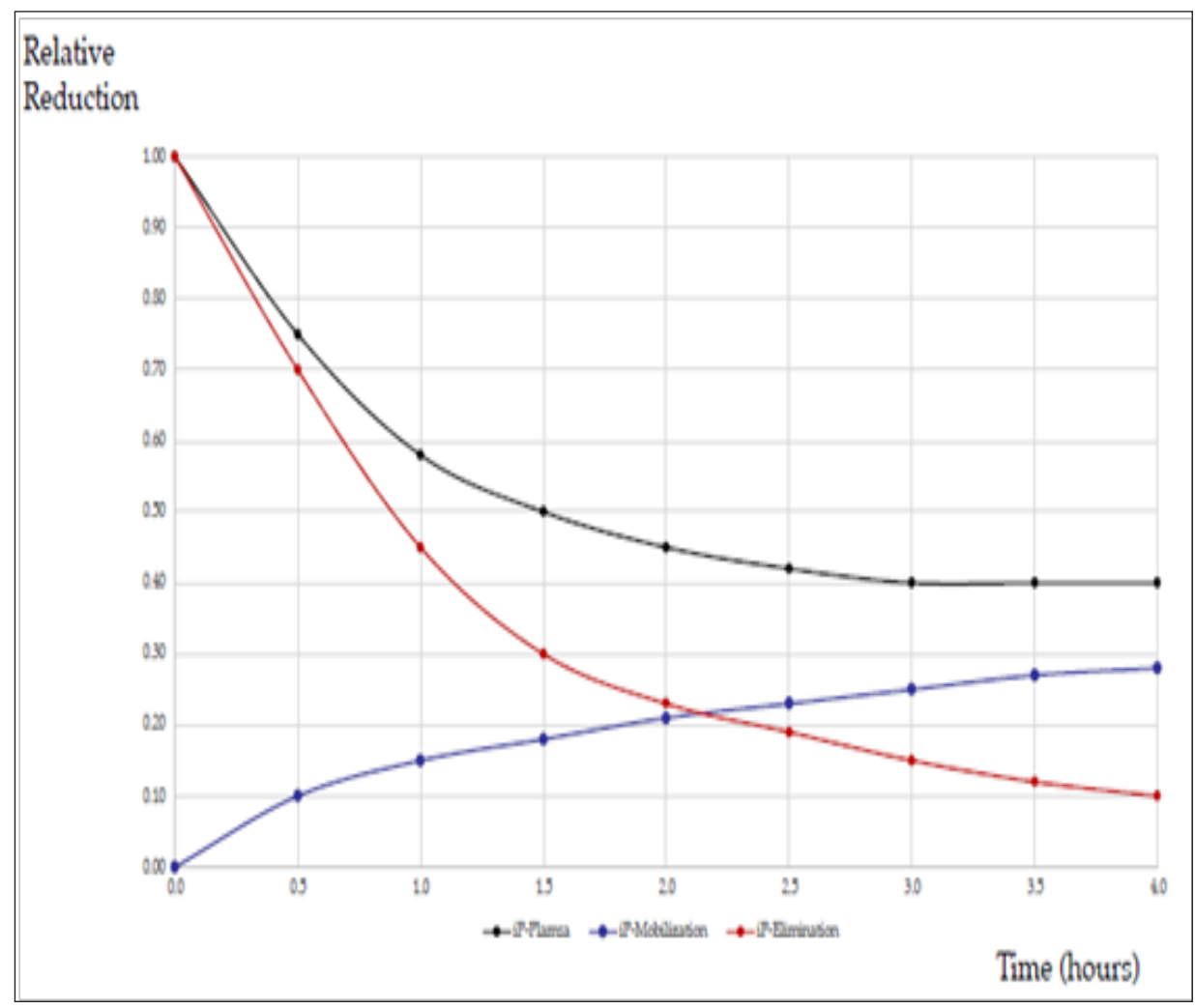

Figure 1: Schematic representation of the 2-phases kinetic profile of serum phosphate during dialysis ( $x$ axis represents time on dialysis; y axis represents the relative reduction of phosphate). The first phase occurs during the first hour of dialysis and it is characterized by a rapid reduction in serum levels while the second phase occurs thereafter, and it is characterized by a plateau in serum concertation. It has been postulated that phosphate mobilization prevents further reductions in serum levels of phosphate while it maintains phosphate removal. Adapted from Kuhlmann Hemodial Int (2006) 10: 338-345 [17]. 
A

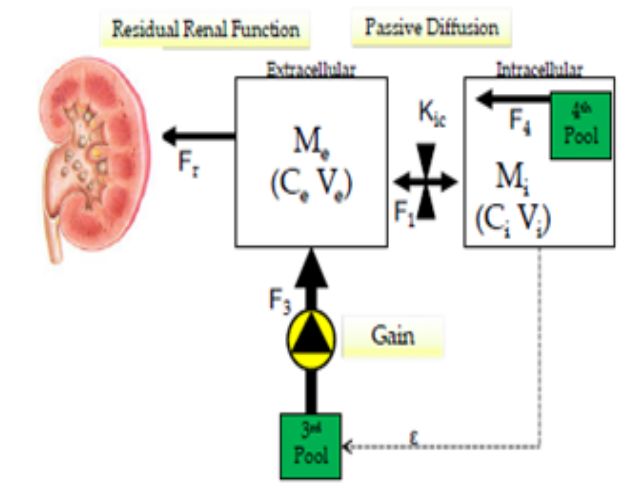

B

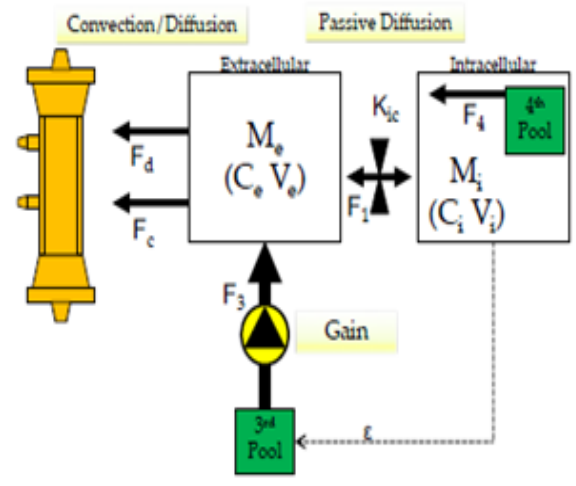

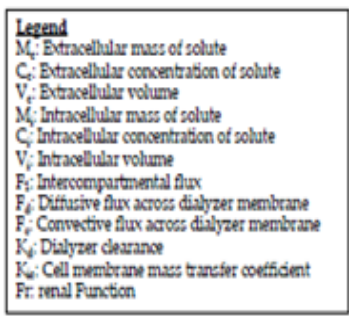

Figure 2: Schematic representation of phosphate 4-pool kinetics. This model summarizes the complicated interplay among intravascular, interstitial and intercellular compartments that influence both phosphate mobilization to and phosphate removal from the intravascular compartment in normal renal function (A) as well as dialysis (B) subjects. Although it is uncleare the exact location, it has been postulated the existence of 2 other pools of phosphate in equilibrium with the extracellular one to compensate for phosphate removal and prevent excessive phosphate concentration in the extracellular and intracellular compartment. Adapted from Kuhlmann Hemodial Int 2006 10: 338-345 [7].

In a cohort of 100 patients with CKD and serum phosphate level of phosphate greater than $6.0 \mathrm{mg} / \mathrm{dl}$, administration of a calcium free vs calcium containing phosphate binder was associated with a significant improvement in endothelial dysfunction [4]. In another small RCT of 239 subjects with CKD stage 3-4 (mean serum phosphate level at study inception $4.8(1.3) \mathrm{mg} / \mathrm{dl}$ ), Di Iorio et al. [19] showed a significant reduction in CKD progression as well as all-cause mortality among patients treated with sevelamer Vs calcium carbonate. While these studies lack of a placebo arm that precludes any definitive conclusion, they show that in subject with sign of phosphate imbalances such as hyperphosphatemia, phosphate modulation in CKD may favorably impact renal as well as allcause survival.In summary, current evidence suggests that chronic exposure to positive phosphate balance especially in the setting of impaired renal function may result in several unwanted effects that ultimately may contribute to the poor survival observed in CKD subjects. Although we lack of reliable markers of phosphate balance and serum levels are only poorly correlated with it, experimental data suggest that treating subjects with overt hyperphosphatemia (i.e. a sign of chronic exposure to positive balance) may impact surrogate as well as hard outcome. Future endeavors are required to identify more accurate biomarkers of phosphate balance as well as to elucidate the role of phosphate control in CKD patient management.

\section{References}

1. Bellasi A (2016) Pro: Should phosphate binders be used in chronic kidney disease stage 3-4? Nephrol Dial Transplant 31(2): 184-188.

2. Bellasi A, Galassi A, Cozzolino M, Biagio Di Iorio (2013) The evolving world of Chronic Kidney Disease Mineral Bone Disorder (CKD-MBD). EMJ Neph 1(1): 20-31.

3. Bellasi A, Cozzolino M, Adragao T,Di Iorio B, Russo D(2013) Phosphate binders in moderate chronic kidney disease: where do we stand? J Nephrol 26(6): 993-1000.

4. Yilmaz MI, Sonmez A, Saglam M, Yaman H, Kilic S, et al. (2012) Comparison of calcium acetate and sevelamer on vascular function and fibroblast growth factor 23 in CKD patients: a randomized clinical trial. Am J Kidney Dis 59(2): 177-185.

5. Ix JH, De Boer IH, Peralta CA, Adeney KL, Duprez DA, et al. (2009) Serum phosphorus concentrations and arterial stiffness among individuals with normal kidney function to moderate kidney disease in MESA. Clin J Am Soc Nephrol 4(3): 609-615.

6. Yamamoto KT, RobinsonCohen C, de Oliveira MC, Alina Kostina, Jennifer A Nettleton, et al. (2013) Dietary phosphorus is associated with greater left ventricular mass. Kidney Int 83: 707-714.

7. Onufrak SJ, Bellasi A, Shaw LJ, Herzog CA, Cardarelli F, et al. (2008) Phosphorus levels are associated with subclinical atherosclerosis in the general population. Atherosclerosis 199(2): 424-431.

8. Russo D, Palmiero G, De Blasio AP, Balletta MM, Andreucci VE, et al. (2004) Coronary artery calcification in patients with CRF not undergoing dialysis. Am J Kidney Dis 44(6): 1024-1030. 
9. Bellasi A, Mandreoli M, Baldrati L,Corradini M, Di Nicolò P, et al. (2011) Chronic Kidney Disease Progression and Outcome According to Serum Phosphorus in Mild-to-Moderate Kidney Dysfunction. Clin J Am Soc Nephrol 6(4): 883-891.

10. Block GA, Klassen PS, Lazarus JM,Ofsthun N, Lowrie EG, et al. (2004) Mineral metabolism, mortality, and morbidity in maintenance hemodialysis. J Am Soc Nephrol 15(8): 2208-2218.

11. Chue CD, Townend JN, Moody WE, Zehnder D, Wall NA, et al. (2013) Cardiovascular Effects of Sevelamer in Stage 3 CKD. J Am Soc Nephrol 24(5): 842-852.

12. Block GA, Wheeler DC, Persky MS, Kestenbaum B, Ketteler M, et al. (2012) Effects of phosphate binders in moderate CKD. J Am Soc Nephrol 23(8): 1407-1415.

13. Dominguez JR, Shlipak MG, Whooley MA, Ix JH (2013) Fractional Excretion of Phosphorus Modifies the Association between Fibroblast Growth Factor-23 and Outcomes. J Am Soc Nephrol 24(4): 647-654.

14. Zoccali C, Ruggenenti P, Perna A, Leonardis D, Tripepi R, et al. (2011) Phosphate may promote CKD progression and attenuate renoprotective effect of ACE inhibition. J Am Soc Nephrol 22(10): 1923-1930.

(C) 9 This work is licensed under Creative

Submission Link: https://biomedres.us/submit-manuscript.php
15. Di Iorio BR, Bellizzi V, Bellasi A, Serena Torraca, Graziella D Arrigo, et al. (2013) Phosphate attenuates the anti-proteinuric effect of very lowprotein diet in CKD patients. Nephrol Dial Transplant 28(3): 632-640.

16. Elias RM, Alvares VRC, Moyses RMA (2018) Phosphate Removal During Conventional Hemodialysis: a Decades-Old Misconception. Kidney Blood Press Res 43(1): 110-114.

17. Kuhlmann MK (2006) Management of hyperphosphatemia. Hemodial Int 10(4): 338-345.

18. Kuhlmann MK (2010) Phosphate elimination in modalities of hemodialysis and peritoneal dialysis. Blood Purif 29(2): 137-144.

19. Di Iorio B, Bellasi A, Russo D (2012) Mortality in kidney disease patients treated with phosphate binders: a randomized study. Clinical journal of the American Society of Nephrology CJASN 7(3): 487-493.

Assets of Publishing with us
BIOMEDICAL
RESEARCHES $\quad \begin{aligned} & \text { Global archiving of articles } \\ & \text { - Immediate, unrestricted online access }\end{aligned}$

